\title{
Electrostatic properties of fullerenes under an external electric field: First-principles calculations of energetics for all IPR isomers from $\mathrm{C}_{60}$ to $\mathrm{C}_{78}$
}

\author{
Jun-ya Sorimachi*, Susumu Okada \\ Graduate School of Pure and Applied Sciences, University of Tsukuba, 1-1-1 Tennodai, \\ Tsukuba, Ibaraki 305-8571, Japan
}

\begin{abstract}
Based on first-principles total energy calculations, we analyze the energetics of the fullerene isomers from $\mathrm{C}_{60}$ to $\mathrm{C}_{78}$, all of which satisfy the isolated pentagon rule, under a parallel electric field. Our calculations show that the total energy of the fullerene is proportional to the square of the external electric field. On the other hand, the coefficient of the quadratic energy profile is sensitive to the fullerene species and their orientation. Furthermore, fullerenes possessing lower symmetry exhibit asymmetric quadratic energy profiles with respect to the field, indicating that they possess intrinsic polarization along particular molecular orientations.
\end{abstract}

Keywords: Fullerene, Electric field, Spherical dielectric, Intrinsic polarization

\section{Introduction}

For the past three decades, fullerenes have maintained a premier position in the fields of nanoscience and nanotechnology as representatives of nanometer-scale materials exhibiting unusual chemical and physical properties not seen in conventional carbon allotropes [1]. Because of the huge number of possible arrangements of 12 pentagonal rings and the appropriate

*TEL/FAX: +81-298535600 (ext. 8233)/+81-298535924

Email addresses: jsorimachi@comas.frsc.tsukuba.ac.jp (Jun-ya Sorimachi), sokada@comas.frsc.tsukuba.ac.jp (Susumu Okada) 
number of hexagonal rings in accordance with Euler's theorem, fullerenes have an infinite number of possible cage structures, from $\mathrm{C}_{20}$ to $\mathrm{C}_{\infty}$, except $\mathrm{C}_{22}[2,3]$. Among these possible fullerenes, $\mathrm{C}_{60}$ is the most abundant fullerene, possessing a truncated icosahedron cage network $[4,5]$. Furthermore, only a limited number of isomers have been extracted from soot among the huge number of possible isomers to date $[6,7,8,9]$. Because of the truncated icosahedron network, $\mathrm{C}_{60}$ possesses highly degenerated states in its $\pi$ electron states, characterized by spherical harmonics $Y_{l m}[10]$. These highly degenerated states can make $\mathrm{C}_{60}$ and its derivatives magnetic [11] and superconducting $[12,13,14,15]$ by injecting the appropriate number of carriers into these states. In addition, other large fullerenes also possess bunched energy levels in their $\pi$ electron states because of the approximately spherical distribution of electrons throughout the networks[10, 16, 17, 18, 19, 20]. On the other hand, early experimental[21, 22, 23, 24, 25] and theoretical[10, $16,17,18,19,20,26,27,28]$ works have demonstrated that the detailed electronic structure of fullerenes sensitively depends on their cage size and network topology.

The approximately spherical electron system makes us wonder whether the fullerene is regarded as a continuum spherical dialectic under an electric field in the classical electrodynamics. It is expected that the bond alternation arising from the arrangement of the pentagons and hexagons in the cage causes the unusual field response that depends on the cage network because of their inhomogeneous charge density. Even though many theoretical and experimental works have elucidated the fundamental properties of fullerenes, fullerenes are still keeping a premier position in emerging materials for the molecular electronic $[29,30]$ and photovoltaic [31] devices in the next generation. In such devices, molecular orientations and bond lengths of fullerenes may affect the resultant device performance. In our previous work, finite length carbon nanotubes showed anomalous field screening against a uniform external electric field induced by the bond alternation [32, 33]. Therefore, in this work, we aim to theoretically investigate the energetics of fullerenes from $\mathrm{C}_{60}$ to $\mathrm{C}_{78}$ (Fig. 1) that satisfy the isolated pentagon rule (IPR) [34, 35] under an external electric field to clarify the influence of the cage network on the external electric field. Using the density functional theory combined with the effective screening medium method, we find that the total energy of fullerene increases with the square of the external field, as in the case of a continuum dielectric within classical electrodynamics. On the other hand, the coefficient of the quadratic curve of the energy is sensitive to the size 
and symmetry of the fullerene. Furthermore, most large fullerenes possess an asymmetric energy profile with respect to the electric field, indicating that these fullerenes possess an intrinsic dipole moment along a particular molecular direction.

\section{Calculation methods and structural models}

All calculations are performed based on density functional theory (DFT) $[36,37]$ as implemented in the program package of the Simulation Tools for Atom TEchnology (STATE) [38]. We use the local density approximation (LDA) $[39,40]$ to treat the exchange correlation potential for interacting electrons. An ultrasoft pseudopotential generated by the Vanderbilt scheme is adopted to describe the interaction between valence electrons and ions [41]. Valence wave functions and charge density are expanded in terms of the plane wave basis set with cutoff energies of 25 and 225 Ry, respectively. $\Gamma$ point sampling is used for Brillouin zone integration. To simulate an isolated fullerene, we considered a large cuboid cell with cell parameters 10, 10, and

$26 \AA$ in the $x, y$, and $z$ directions, respectively. To study the behavior of the fullerene in a uniform electric field, we adopted the effective screening medium method (ESM) to solve the Poisson's equation, including the external electric field [42]. We applied a parallel electric field from -25.7 to $25.7 \mathrm{mV} / \AA$ between two metal electrodes, simulated by an effective screening medium with an infinite permittivity; the electrodes are situated at the cell boundaries along the $z$ direction, $z= \pm 13 \AA$ (Fig. 2). In the present study, we have investigated the energetics of all IPR isomers up to $\mathrm{C}_{78}$, each of which possesses seven representative molecular arrangements with respect to the electric field $\left(\theta=0,15,30,45,60,75\right.$, and $\left.90^{\circ}\right)$ (Fig. 2). The geometric structures of the fullerenes are fully optimized until the remaining force acting on each atom is less than $5 \mathrm{mRy} / \AA$. During calculations under an electric field up to $\pm 25.7 \mathrm{mV} / \AA$, the geometric structure of the fullerenes is fixed to that under zero electric field because the field is too small to cause a force inducing the structural reconstruction.

\section{Results and discussion}

Figure 3 shows the relative energy of fullerenes with the molecular orientations $\theta=0^{\circ}, 45^{\circ}$, and $90^{\circ}$ with respect to the electric field as a function of the field. The energies are calculated from that of the fullerenes without 

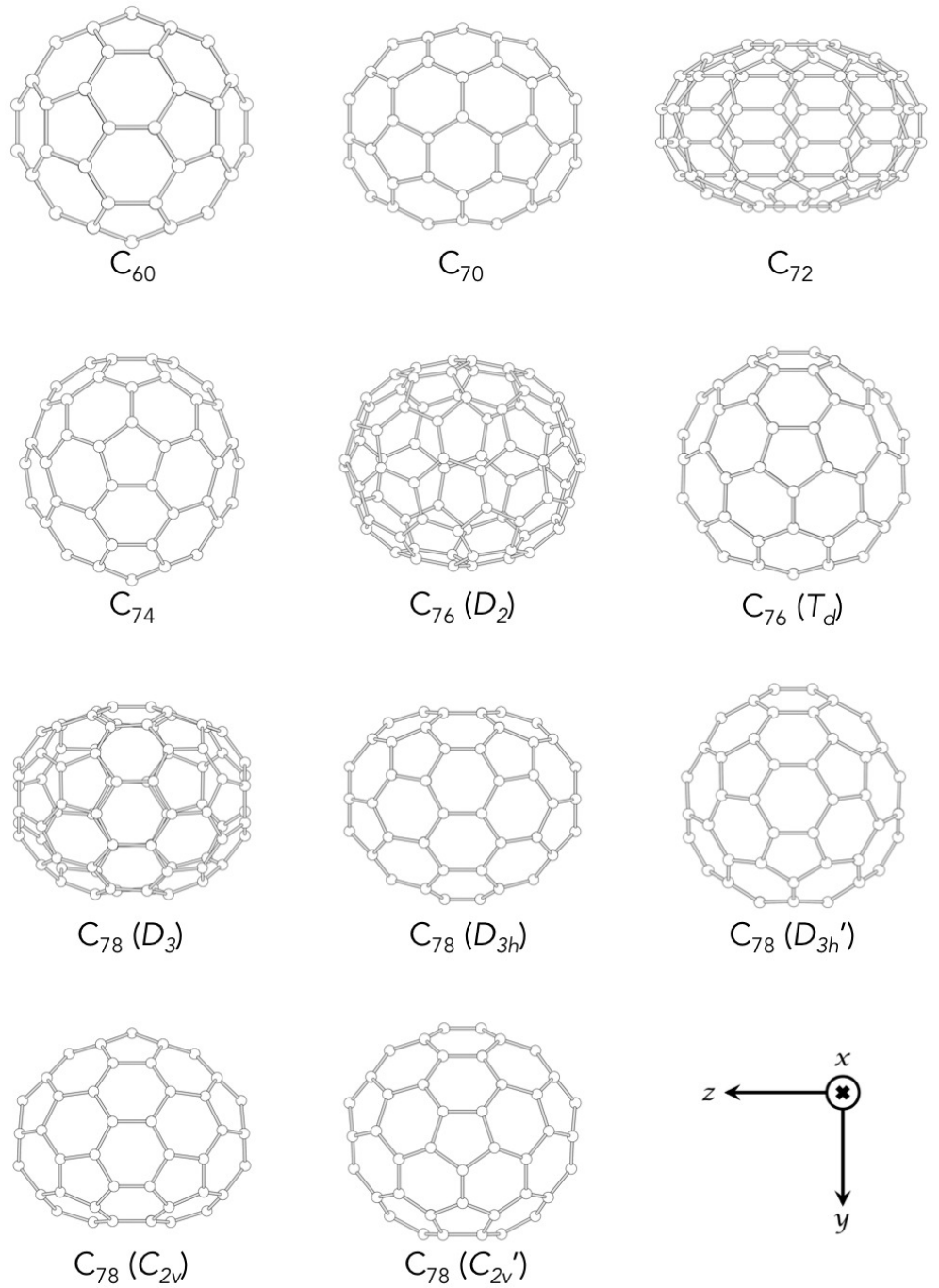

Figure 1: Optimized geometries of all IPR isomers up to $\mathrm{C}_{78}$. These correspond to the molecular arrangements $\theta=0^{\circ}$ defined in Fig. 2. 


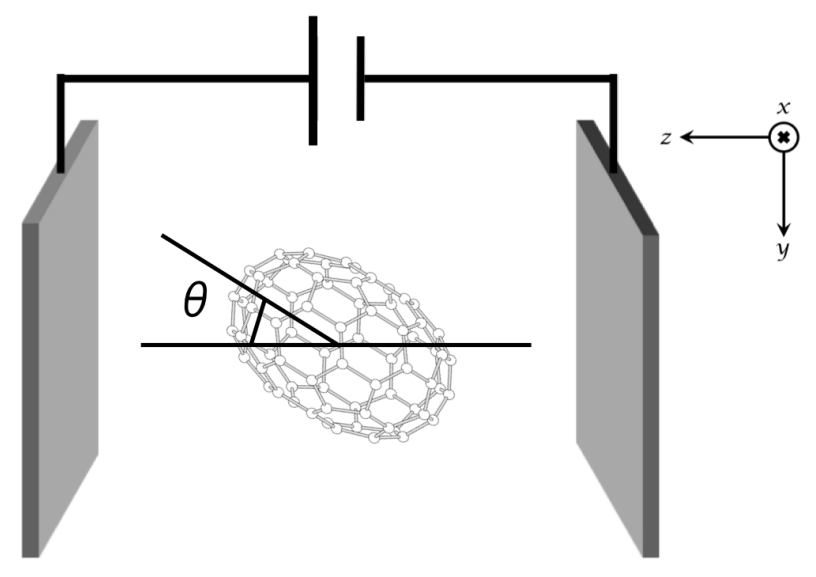

Figure 2: A structural model of fullerenes under an electric field. Gray slabs represent the parallel electrodes simulated by an effective screening medium with infinite relative permittivity. The molecular arrangement is defined by the molecular angle $\theta$ with respect to the electric field.

the electric field. The relative energy of the fullerenes is proportional to the square of the electric field. The quadratic behavior is qualitatively the same as the energy profile of a spherical dielectric under a uniform electric field. This fact indicates that fullerenes naively behave as a spherical dielectric under an electric field. In particular, for $\mathrm{C}_{60}$, the quadratic energy curve with respect to the electric field is independent of the molecular arrangement, indicating that the spherical shell model is applicable for describing the energetics of $\mathrm{C}_{60}$ under an electric field.

For $\mathrm{C}_{70}, \mathrm{C}_{72}, \mathrm{C}_{76}\left(D_{2}\right)$, and $\mathrm{C}_{78}\left(D_{3}\right)$, the total energy also exhibits quadratic and symmetric behavior with respect to the energy minimum at zero electric field. However, in contrast to $\mathrm{C}_{60}$, the coefficient of the quadratic curve depends on the relative orientation of the fullerene to the electric field because of their characteristic cage structure, e.g., ellipsoidal and flat shapes for $\mathrm{C}_{70}$ and $\mathrm{C}_{72}$ respectively. Therefore, it seems to be necessary to make a geometrical correction in the continuum spherical model to describe the energetics of these fullerenes under an electric field.

The remaining fullerenes (i.e. $\mathrm{C}_{74}, \mathrm{C}_{76}\left(T_{d}\right), \mathrm{C}_{78}\left(D_{3 h}\right), \mathrm{C}_{78}\left(D_{3 h}{ }^{\prime}\right), \mathrm{C}_{78}\left(C_{2 v}\right)$, and $\left.\mathrm{C}_{78}\left(C_{2 v}{ }^{\prime}\right)\right)$ exhibit anomalous behavior with respect to the electric field: the total energy of the fullerene with a particular molecular arrangement is lower than that under zero electric field. In particular, two of five $\mathrm{C}_{78}$, i.e. 

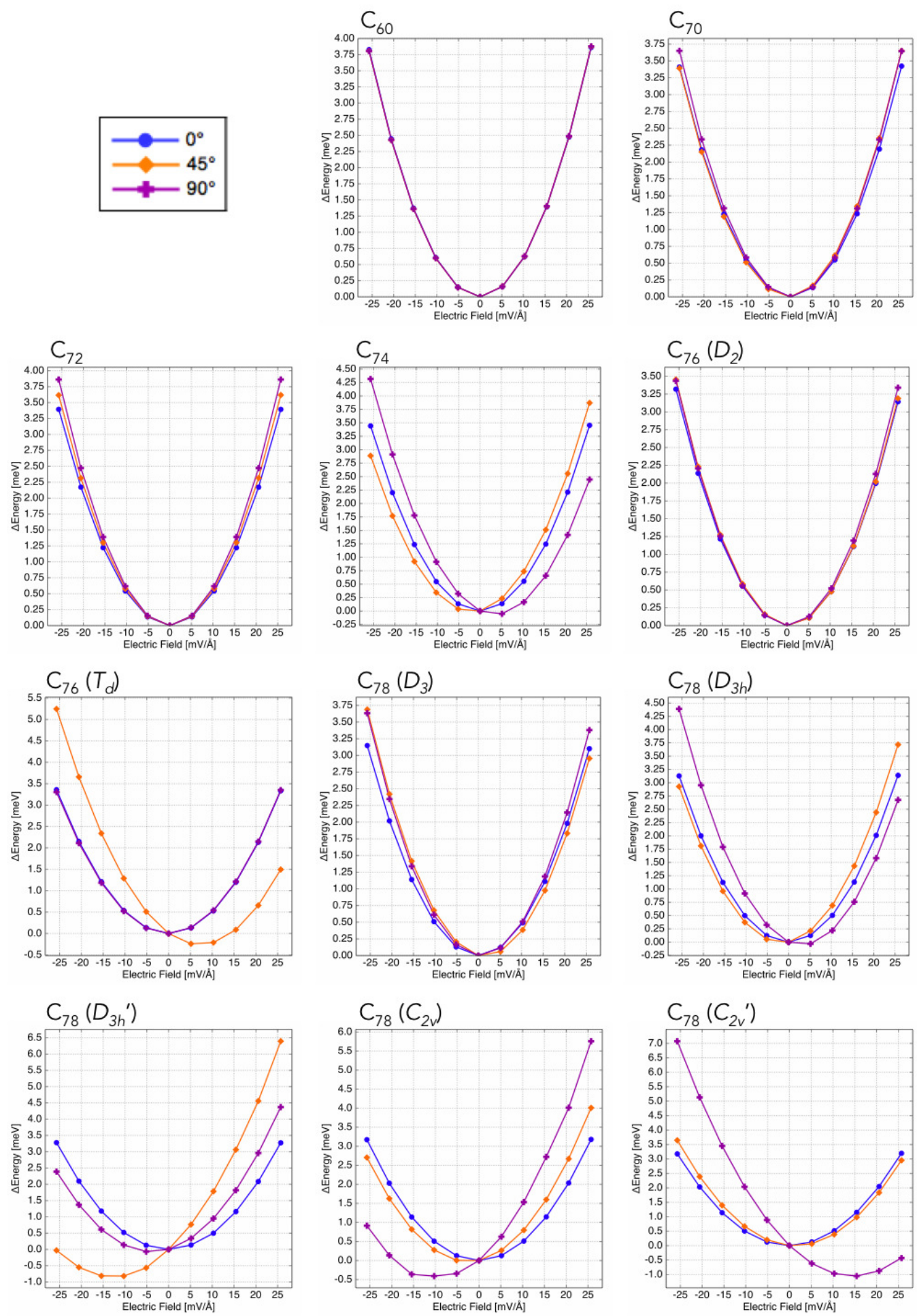

Figure 3: Relative energies of fullerenes with molecular orientations $\theta=0,45$, and $90^{\circ}$ as a function of the electric field. Energies are measured from the energy of the fullerenes without an electric field. 
$D_{3 h}$ ' and $C_{2 v}$ ' isomers with angles $\theta=45^{\circ}$ and $90^{\circ}$, respectively, have considerably lower total energy under an electric field of approximately $15 \mathrm{mV} / \AA$. This fact indicates that these fullerenes possess a dipole moment along the direction of the field under these molecular angles because the total energy decreases by the scalar product of the dipole moment and electric field. The dipole moment arises from the arrangement of pentagons in the cage. For $\mathrm{C}_{78}\left(C_{2 v}{ }^{\prime}\right)$ with the molecular arrangement of $\theta=90^{\circ}$, eight of twelve pentagons concentrate at the upper hemisphere of the cage. Because of the single bond nature of the pentagons, the charge density in the upper hemisphere is lower than that in the other hemisphere, leading to an asymmetric charge density distribution between the two hemispheres of $\mathrm{C}_{78}\left(C_{2 v}{ }^{\prime}\right)$.

The total energy of a fullerene under an electric field has the formula $E_{t o t}=\alpha|\boldsymbol{E}|^{2}-\boldsymbol{d} \cdot \boldsymbol{E}$, where $\boldsymbol{E}$ and $\boldsymbol{d}$ are the electric field and dipole moment of the fullerene, respectively. To enable a quantitative discussion of the energetics, we summarize the coefficient $\alpha$ of fullerenes with various molecular orientations (Table 1). For $\mathrm{C}_{60}$, the coefficient is exactly the same for all molecular angles, within numerical error. This result corroborates the fact that the electrons of $\mathrm{C}_{60}$ can be regarded as a spherical shell with 7 $\AA$ diameter. The coefficient of the other fullerenes strongly depends on the molecular orientation. For $\mathrm{C}_{70}$ and $\mathrm{C}_{72}$, the coefficient under the molecular angle $\theta=90^{\circ}$ is larger than that under $\theta=0^{\circ}$. The increase in the coefficient under $\theta=90^{\circ}$ is ascribed to the arrangement of the hexagons with respect to the electric field, which screen the field much more than the pentagons do because of the higher charge density in their covalent bonds. In both fullerenes, the hexagon-rich area is arranged at the electrode side under the angle $\theta=90^{\circ}$.

Finally, we discuss the asymmetric energy profile with respect to the electric field for $\mathrm{C}_{74}, \mathrm{C}_{76}\left(T_{d}\right), \mathrm{C}_{78}\left(D_{3 h}{ }^{\prime}\right), \mathrm{C}_{78}\left(C_{2 v}\right)$, and $\mathrm{C}_{78}\left(C_{2 v}{ }^{\prime}\right)$ fullerenes. The asymmetric profile implies that these fullerenes inherently possess polarization along a particular molecular orientation. Table 2 summarizes the internal electric field induced by the intrinsic polarization of the fullerene cage along the $z$ direction with molecular orientations ranging from 0 to $90^{\circ}$. Fullerenes with a symmetric energy profile with respect to the field do not have an intrinsic electric field. On the other hand, $\mathrm{C}_{74}, \mathrm{C}_{76}\left(T_{d}\right), \mathrm{C}_{78}\left(D_{3 h}{ }^{\prime}\right)$, $\mathrm{C}_{78}\left(C_{2 v}\right)$, and $\mathrm{C}_{78}\left(C_{2 v}{ }^{\prime}\right)$ fullerenes have an intrinsic electric field of approximately $10 \mathrm{mV}$, which complexly depends on the molecular orientation. This complex orientation dependence may arise from the relative arrangement of pentagons in fullerene cages because of the lower bond charge density, as 
Table 1: The coefficient of the square of the electric field for each fullerene.

\begin{tabular}{lccccccc}
\hline \multicolumn{7}{c}{ Coefficient $\alpha\left[\mathrm{eV}^{2} / \mathrm{V}^{2}\right]$} \\
\hline fullerene & $0^{\circ}$ & $15^{\circ}$ & $30^{\circ}$ & $45^{\circ}$ & $60^{\circ}$ & $75^{\circ}$ & $90^{\circ}$ \\
\hline $\mathrm{C}_{60}$ & 5.8 & 5.8 & 5.8 & 5.8 & 5.8 & 5.8 & 5.8 \\
$\mathrm{C}_{70}$ & 5.2 & 5.2 & 5.2 & 5.3 & 5.4 & 5.5 & 5.5 \\
$\mathrm{C}_{72}$ & 5.1 & 5.2 & 5.3 & 5.5 & 5.6 & 5.8 & 5.8 \\
$\mathrm{C}_{74}$ & 5.2 & 5.1 & 5.1 & 5.1 & 5.1 & 5.1 & 5.1 \\
$\mathrm{C}_{76}\left(D_{2}\right)$ & 4.9 & 4.9 & 4.9 & 5.0 & 5.1 & 5.1 & 5.1 \\
$\mathrm{C}_{76}\left(T_{d}\right)$ & 5.1 & 5.0 & 5.1 & 5.1 & 5.1 & 5.1 & 5.0 \\
$\mathrm{C}_{78}\left(D_{3}\right)$ & 4.7 & 4.8 & 4.9 & 5.0 & 5.2 & 5.2 & 5.3 \\
$\mathrm{C}_{78}\left(D_{3 h}\right)$ & 4.7 & 4.8 & 4.9 & 5.0 & 5.2 & 5.2 & 5.3 \\
$\mathrm{C}_{78}\left(D_{3 h}{ }^{\prime}\right)$ & 5.0 & 4.9 & 5.1 & 4.8 & 5.0 & 4.8 & 5.1 \\
$\mathrm{C}_{78}\left(C_{2 v}\right)$ & 4.8 & 4.8 & 5.0 & 5.1 & 5.2 & 5.3 & 5.0 \\
$\mathrm{C}_{78}\left(C_{2 v}{ }^{\prime}\right)$ & 4.8 & 4.8 & 4.9 & 5.0 & 5.1 & 5.1 & 5.0 \\
\hline
\end{tabular}

discussed above.

\section{Summary}

Using DFT combined with the ESM method, we have investigated the energetics of all IPR isomers up to $\mathrm{C}_{78}$ under an external electric field. DFTESM calculations reveal that the total energy of the fullerenes increases with the square of the external field. On the other hand, the coefficient of the quadratic energy curve is sensitive to the size, symmetry, and orientation of the fullerene. In the case of $\mathrm{C}_{60}$, we find a perfectly symmetrical quadratic profile, indicating that the electron system of $\mathrm{C}_{60}$ can be regarded as a spherical dielectric in classical electrodynamics. For large fullerenes possessing higher symmetry, such as $\mathrm{C}_{70}$ and $\mathrm{C}_{72}$, the coefficient depends on the mutual orientation of the fullerenes with respect to the field. Furthermore, for fullerenes with lower symmetry, such as $\mathrm{C}_{74}, \mathrm{C}_{78}\left(C_{2 v}\right)$, and $\mathrm{C}_{78}\left(C_{2 v}{ }^{\prime}\right)$, the quadratic energy curve shows an asymmetric nature with respect to the electric field and has an energy minimum at a non-zero electric field, indicating that these fullerenes possess polarization along the molecular axis. These unusual features of the energetics are ascribed to the inhomogeneity of the charge density on the fullerene cage arising from the bond alternation caused by the pentagonal rings. 
Table 2: The intrinsic internal electric field of all IPR fullerene isomers up to $\mathrm{C}_{78}$ with orientation angles of $0,15,30,45,60,75$, and $90^{\circ}$ along the $z$ axis.

\begin{tabular}{lccccccc}
\hline \multicolumn{7}{c}{ Internal electric field $\left[10^{-3} \mathrm{~V} / \AA\right]$} \\
\hline fullerene & $0^{\circ}$ & $15^{\circ}$ & $30^{\circ}$ & $45^{\circ}$ & $60^{\circ}$ & $75^{\circ}$ & $90^{\circ}$ \\
\hline $\mathrm{C}_{60}$ & -0.10 & -0.40 & -0.18 & -0.26 & -0.26 & -0.27 & -0.12 \\
$\mathrm{C}_{70}$ & 0.01 & -0.17 & -0.14 & -0.81 & -3.61 & -0.68 & 0.09 \\
$\mathrm{C}_{72}$ & 0.01 & -0.12 & -0.09 & 0.06 & -0.15 & 0.18 & -0.28 \\
$\mathrm{C}_{74}$ & 0.37 & 14.92 & -16.65 & -1.67 & 8.04 & -5.23 & 4.64 \\
$\mathrm{C}_{76}\left(D_{2}\right)$ & 0.50 & -0.35 & -0.04 & 0.68 & 0.67 & 0.09 & 0.38 \\
$\mathrm{C}_{76}\left(T_{d}\right)$ & -0.15 & -1.22 & -2.19 & 11.97 & 4.01 & 4.98 & -0.31 \\
$\mathrm{C}_{78}\left(D_{3}\right)$ & 0.11 & -0.63 & 0.60 & 2.07 & 2.18 & -0.48 & 0.61 \\
$\mathrm{C}_{78}\left(D_{3 h}\right)$ & -0.44 & 1.31 & -2.37 & -1.66 & 1.14 & 4.75 & 2.45 \\
$\mathrm{C}_{78}\left(D_{3 h}{ }^{\prime}\right)$ & -0.18 & -14.87 & 5.25 & -15.08 & -0.30 & 13.36 & -6.65 \\
$\mathrm{C}_{78}\left(C_{2 v}\right)$ & 0.16 & -2.05 & 0.28 & -2.99 & -1.52 & -15.83 & -15.42 \\
$\mathrm{C}_{78}\left(C_{2 v}\right)$ & 0.01 & -0.09 & 8.28 & 1.18 & 8.38 & -6.76 & 18.35 \\
\hline
\end{tabular}

\section{Acknowledgements}

This work was supported by CREST, from the Japan Science and Technology Agency, a Grant-in-Aid for Scientific Research from the Ministry of Education, Culture, Sports, Science and Technology of Japan, and the Joint Research Program on Zero-Emission Energy Research, Institute of Advanced Energy, Kyoto University. A part of the calculations was performed on an NEC SX-Ace at the Cybermedia Center at Osaka University and on an NEC SX-9 at the Institute of Solid State Physics, The University of Tokyo.

[1] M.S. Dresselhaus, G. Dresselhaus, P.C. Eklund, Science of Fullerenes and Carbon Nanotubes (Academic Press, San Diego, CA, 1996).

[2] P.W. Fowler, D.E. Manolopoulos, An Atlas of Fullerenes (Oxford University Press, Oxford, U. K., 1995).

[3] D.E. Manolopoulos, P.W. Fowler, J. Chem. Phys. 96 (1992) 7603.

[4] H.W. Kroto, J.R. Heath, S.C. O'Brien, R.F. Curl, R.E. Smalley, Nature 318 (1985) 162.

[5] E.A. Rohlfing, D.M. Cox, A. Kaldor, J. Chem. Phys. 81 (1984) 3322. 
[6] R. Ettl, I. Chao, F. Diederich, R.L. Whetten, Nature 353 (1991) 149.

[7] F. Diederich, R. Ettle, Y. Rubin, R.L. Whetten, R. Beck, M. Alvarez, S. Anz, D. Shensharma, F. Wudl, K.C. Khemani, A. Koch, Science 252 (1991) 548.

[8] F. Diederich, R.L. Whetten, C. Thulgen, R. Ettle, I. Chao, M. Alvarez, Science 254 (1991) 1768.

[9] K. Kikuchi, N. Nakahara, T. Wakabayashi, S. Suzuki, H. Shiromaru, Y. Miyake, K. Saito, I. Ikemoto, M. Kainosho, Y. Achiba, Nature 357 (1992) 142 .

[10] S. Saito, A. Oshiyama, Phys. Rev. Lett. 66 (1991) 2637.

[11] P.-M. Allemand, K.C. Khemani, A. Koch, F. Wuld, K. Holczer, S. Donovan, G. Gruner, J.D. Thompson, Science, 253 (1991) 301.

[12] A.F. Hebard, M.J. Rosseinsky, R.C. Haddon, D.W. Murphy, S.H. Glarum, T.T.M. Palstra, A.P. Ramirez, A.R. Kortan, Nature 350 (1991) 600 .

[13] M.J. Rosseinsky, A.P. Ramirez, S.H. Glarum, D.W. Murphy, R.C. Haddon, A.F. Hebard, T.T.M. Palstra, A.R. Kortan, S.M. Zahurak, A.V. Makhija, Phys. Rev. Lett. 66 (1991) 2830.

[14] P.W. Stephens, L. Mihaly, P.L. Lee, R.L. Whetten, S.-M Huang, R. Kaner, F. Deiderich, K. Holczer, Nature 351 (1991) 632.

[15] K. Tanigaki, T.W. Ebbesen, S. Saito, J. Mizuki, J.S. Tsai, Y. Kubo, S. Kuroshima, Nature 352 (1991) 222.

[16] S. Saito, A. Oshiyama, Phys. Rev. B 44 (1991) 11532(R).

[17] J.R. Colt, G.E. Scuseria, J. Phys. Chem. 96 (1992) 10265.

[18] K. Nakao, N. Kurita, M. Fujita, Phys. Rev. B 49 (1994) 11415.

[19] S. Saito, S. Okada, S. Sawada, N. Hamada, Phys. Rev. Lett. 75 (1995) 685 .

[20] S. Okada, S. Saito, Chem. Phys. Lett. 252 (1996) 94. 
[21] S. Hino, H. Takahashi, K. Iwasaki, T. Miyazaki, K. Kikuchi, Y. Achiba, Chem. Phys. Lett., 230 (1994) 165.

[22] S. Hino, K. Matsumoto, S. Hasegawa, K. Kamiya, H. Inokuchi, T. Morikawa, T. Takahashi, K. Seki, K. Kikuchi, S. Suzuki, I. Ikemoto, Y. Achiba, Chem. Phys. Lett. 190 (1992) 169.

[23] S. Hino, K. Matsumoto, S. Hasegawa, K. Iwasaki, K. Yakushi, T. Morikawa, T. Takahashi, K. Seki, K. Kikuchi, S. Suzuki, I. Ikemoto, Y. Achiba, Phys. Rev. B 48 (1993) 8418.

[24] S. Hino, K. Umishita, K. Iwasaki, T. Miyazaki, K. Kikuchi, Y. Achiba, Phys. Rev. B 53 (1996) 7496.

[25] S. Hino, K. Matsumoto, S. Hasegawa, H. Inokuchi, T. Morikawa, T. Takahashi, K. Seki, K. Kikuchi, S. Suzuki, I. Ikemoto, Y. Achiba, Chem. Phys. Lett. 197 (1992) 38.

[26] B.L. Zhang, C.Z. Wang, K.M. Ho, Chem. Phys. Lett, 193 (1992) 225.

[27] B.L. Zhang, C.Z. Wang, K. M. Ho, C.H. Xu, C.T. Chan, J. Chem. Phys. 98 (1993) 3095.

[28] X.Q. Wang, C.Z. Wang, B.L. Zhang, K.M. Ho, Z. Phys. D, 26 (1993) 264.

[29] J. Zaumseil and H. Sirringhaus, Chem. Rev. 107 ( 2007) 1296.

[30] S. Kobayashi, T. Nishikawa, T. Takenobu, S. Mori, T. Shimoda, T. Mitani, H. Shimotani, N. Yoshimoto, S. Ogawa, and Y. Iwasa, Nat. Mater. 3 (2004) 317.

[31] Y. Matsuo, Y. Sato, T. Niinomi, I. Soga, H. Tanaka, and E. Nakamura, J. Am. Chem. Soc. 131 (2009) 16048.

[32] A. Yamanaka, S. Okada, Appl. Phys. Express 5 (2012) 095101.

[33] A. Yamanaka, S. Okada, Appl. Phys. Express 6 (2013) 045101.

[34] T.G. Schmalz, W.A. Seitz, D.J. Klein, G.E. Hite, Chem. Phys. Lett. 130 (1986) 203. 
[35] H.W. Kroto, Nature 329 (1987) 529.

[36] P. Hohenberg, W. Kohn, Phys. Rev. 136 (1964) B864.

[37] W. Kohn, L.J. Sham, Phys. Rev. 140 (1965) A1133.

[38] Y. Morikawa, K. Iwata, K. Terakura, Appl. Surf. Sci. 169-170 (2001) 11.

[39] J.P. Perdew, A. Zunger, Phys. Rev. B 23 (1981) 5048.

[40] D.M. Ceperley, B.J. Alder, Phys. Rev. Lett. 45 (1980) 566.

[41] D. Vanderbilt, Phys. Rev. B 41 (1990) 7892.

[42] M. Otani, O. Sugino, Phys. Rev. B 73 (2006) 115407. 
Graphical Abstract

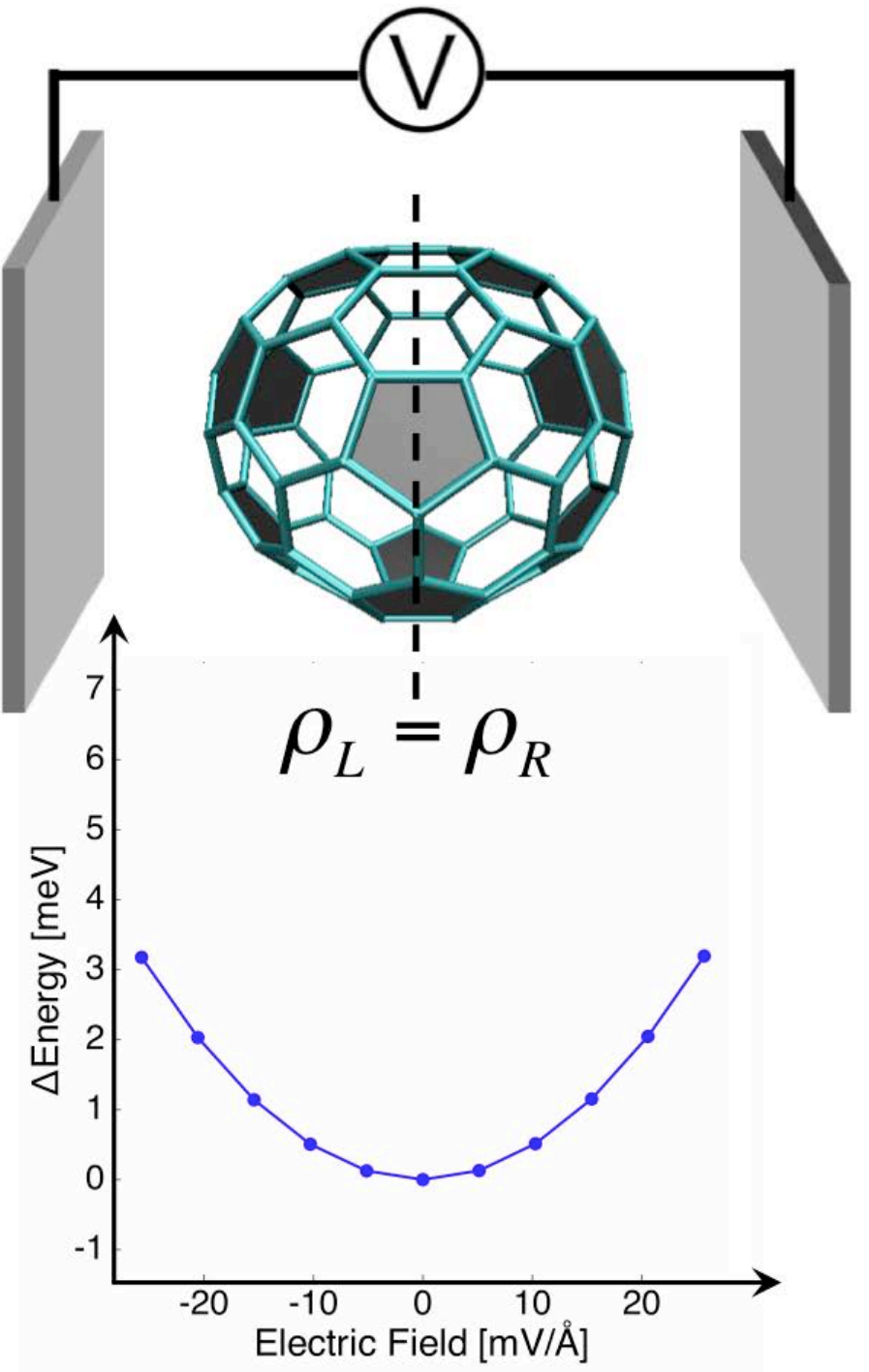

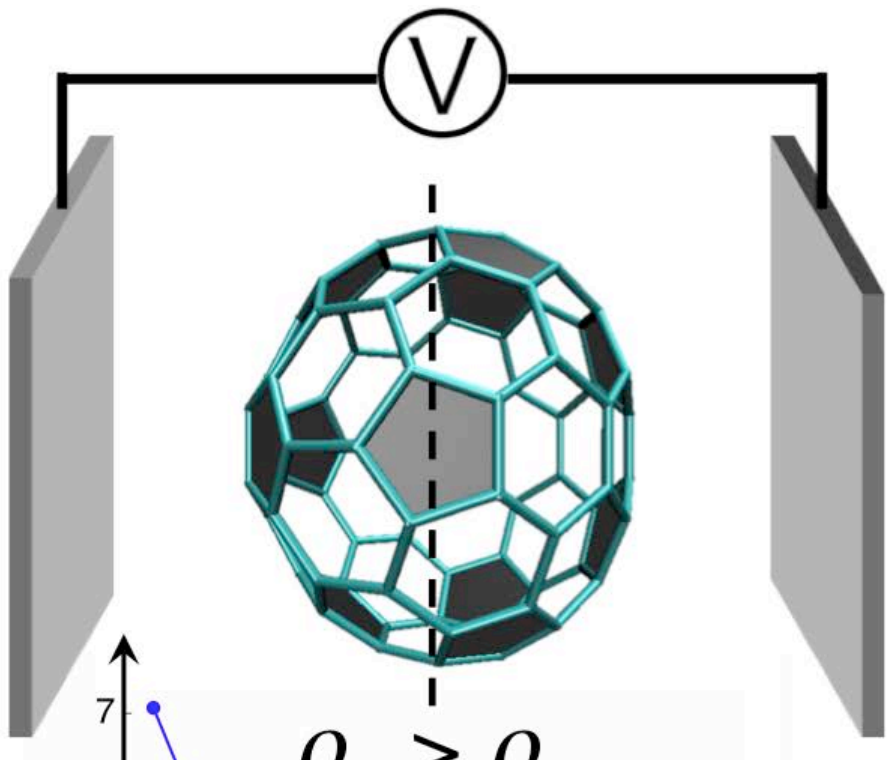

